\title{
On a quantitative refinement of the Lagrange spectrum
}

\author{
by
}

\author{
Edward B. Burger, Amanda Folsom, Alexander Pekker, \\ Rungporn Roengpitya and Julia Snyder (Williamstown, MA)
}

1. Introduction. Let $\alpha$ be an irrational real number. In 1879 Markoff [6], using the theory of quadratic forms, proved that there exist infinitely many positive integers $q$ satisfying the inequality

$$
q\|\alpha q\| \leq 1 / \sqrt{5},
$$

where $\|\cdot\|$ denotes the distance to the nearest integer function. Furthermore, the constant $1 / \sqrt{5}$ is best possible. Twelve years later, Hurwitz [5] offered a more direct argument and as a consequence this well-known result often bears his name.

While we carefully define all objects and notation in the following two sections, here we briefly review Markoff's fundamental observations. If we consider only those irrational $\alpha$ for which inequality (1.1) is not best possible, then (1.1) can be replaced by the sharper inequality

$$
q\|\alpha q\| \leq 1 / \sqrt{8},
$$

and this inequality cannot be improved for certain $\alpha$. Repeating this process we generate a sequence of best possible constants which comprise the smallest values of the Lagrange spectrum. This sequence, which begins with $\mu_{1}=\sqrt{5}, \mu_{2}=\sqrt{8}, \mu_{3}=\sqrt{221} / 5, \mu_{4}=\sqrt{1517} / 13$, was explicitly given by Markoff in terms of the sequence of integers known as Markoff numbers $m_{1}, m_{2}, \ldots$ arising from integer solutions to the diophantine equation

$$
x^{2}+y^{2}+z^{2}=3 x y z .
$$

In particular,

$$
\mu_{r}=\frac{\sqrt{9 m_{r}^{2}-4}}{m_{r}} .
$$

Moreover the constant $\mu_{r}$ is best possible for any $\alpha$ equivalent to $\alpha_{m_{r}}$ as defined in (3.2). In particular, we note that $\alpha_{m_{1}}=(-1+\sqrt{5}) / 2$ and $\alpha_{m_{2}}=$

2000 Mathematics Subject Classification: 11J06, 11J25, 11J70. 
$-1+\sqrt{2}$. It follows from (1.2) that the smallest accumulation point of the Lagrange spectrum is 3 .

In 1947, Davenport asked for the best constant when only a fixed, finite number of solutions to an inequality of the form (1.1) is desired. The answer was quickly found by Prasad [7] (see also [9]) and is given here as

Theorem 1.1. For $n=1,2, \ldots$, let

$$
C_{1}(n)=\frac{1+\sqrt{5}}{2}+\frac{p_{2 n-1}}{q_{2 n-1}}
$$

where $p_{l} / q_{l}$ denotes the lth convergent of $(-1+\sqrt{5}) / 2$. Then for any irrational number $\alpha$, the inequality

$$
q\|\alpha q\| \leq 1 / C_{1}(n)
$$

has at least $n$ positive integer solutions $q$. Moreover, for any $n$, the constant $C_{1}(n)$ cannot be improved when $\alpha=(-1+\sqrt{5}) / 2$.

Theorem 1.1 inspired the problem of finding the best possible constants $C_{2}(n)$ for those $\alpha$ not equivalent to $\alpha_{m_{1}}$. These constants were found by Eggan [4] in 1961. In particular he showed that

$$
C_{2}(n)=1+\sqrt{2}+\frac{p_{2 n-1}}{q_{2 n-1}},
$$

where $p_{l} / q_{l}$ denotes the $l$ th convergent of $-1+\sqrt{2}$. These constants are best possible for $\alpha=-1+\sqrt{2}$. In 1971, Prasad and Prasad [8] showed that

$$
C_{3}(n)=\frac{11+\sqrt{221}}{10}+\frac{p_{4 n-1}}{q_{4 n-1}},
$$

where $p_{l} / q_{l}$ denotes the $l$ th convergent of $\alpha_{m_{3}}$. These constants are best possible for $\alpha=\alpha_{m_{3}}=(-11+\sqrt{221}) / 10$.

While for the first three values of $r$, the constant $C_{r}(n)$ equals the sum of $-\bar{\alpha}_{m_{r}}$ and a convergent of $\alpha_{m_{r}}$, the question of finding an explicit formulation for $C_{r}(n)$ in general remained open. It is clear from the definition of $C_{r}(n)$ that

$$
\lim _{n \rightarrow \infty} C_{r}(n)=\mu_{r} .
$$

However, while previous results give the best possible constant for all $n$ in the cases $r=1,2$, and 3 , a question of greater interest is to explicitly find, for example, $C_{r}(1)$ for $r=1,2, \ldots$ This sequence would be the analogue of the Lagrange spectrum in the case when only one solution is desired. Clearly, finding the first accumulation point of this new spectrum as $r \rightarrow \infty$ would be of interest.

In this paper we resolve all these issues by explicitly computing $C_{r}(n)$ for arbitrary $r$ and $n$. While we can define the values $C_{r}(n)$ in a manner sympathetic to the previously known values, in practice, it may be awkward 
to have the convergents appearing in the formulation. Thus we first provide a definition for $C_{r}(n)$ that depends only on $n$ and the $r$ th Markoff number $m_{r}$. In this direction, for a fixed, positive integer $r$, we define the recurrence sequence $\mathcal{Z}_{r}(n)$ by $\mathcal{Z}_{r}(0)=0, \mathcal{Z}_{r}(1)=1$, and for $n>1$,

$$
\mathcal{Z}_{r}(n)=3 m_{r} \mathcal{Z}_{r}(n-1)-\mathcal{Z}_{r}(n-2) .
$$

We may now state our main result as

TheOREM 1.2. For positive integers $r$ and $n$,

$$
C_{r}(n)=\frac{\sqrt{9 m_{r}^{2}-4}}{2 m_{r}}+\frac{3}{2}-\frac{\mathcal{Z}_{r}(n-1)}{m_{r} \mathcal{Z}_{r}(n)} .
$$

More precisely, for an irrational number $\alpha$ not equivalent to $\alpha_{m_{s}}$ for any $s$, $s<r$, the inequality

$$
q\|\alpha q\| \leq 1 / C_{r}(n)
$$

has at least $n$ positive integer solutions $q$. Moreover, the constant $C_{r}(n)$ is best possible for $\alpha=\alpha_{m_{r}}$.

From (1.2), it immediately follows that

$$
C_{r}(n)=\frac{3+\mu_{r}}{2}-\frac{\mathcal{Z}_{r}(n-1)}{m_{r} \mathcal{Z}_{r}(n)} .
$$

In particular, we see that for all $r$,

$$
C_{r}(1)=\left(3+\mu_{r}\right) / 2 .
$$

It also follows from Lemma 4.2 that for any fixed $r \geq 1, C_{r}(n)$ is decreasing as a function of $n$, and

$$
\lim _{n \rightarrow \infty} C_{r}(n)=\mu_{r}
$$

Hence the explicit formulation of $C_{r}(n)$ given in Theorem 1.2 provides a quantitative version of Markoff's results on the Lagrange spectrum.

Furthermore, another application of Lemma 4.2 allows us to deduce the following interesting corollary.

Corollary 1.3. Let $C_{r}(n)$ be as defined in Theorem 1.2. Then

$$
\lim _{r \rightarrow \infty} C_{r}(n)=3 .
$$

Thus we conclude that for any fixed $n$, the first accumulation point in the spectrum of best possible constants for which there are at least $n$ solutions to the associated diophantine inequality is equal to the first accumulation point of the best possible constants that ensure infinitely many solutions.

As will be shown in Section 4, we can also express $C_{r}(n)$ in terms of $\alpha_{m_{r}}$ and its convergents, thus producing a formulation that is more in sympathy with previous results. 
Theorem 1.4. Let $C_{r}(n)$ be as defined in Theorem 1.2. For $r=1$ and $r=2$, let $L=2$, and for $r \geq 3$, let $L$ denote the smallest period length of the continued fraction expansion for $\alpha_{m_{r}}$. Then

$$
C_{r}(n)=-\bar{\alpha}_{m_{r}}+\frac{p_{n L-1}}{q_{n L-1}},
$$

where $p_{l} / q_{l}$ denotes the lth convergent of $\alpha_{m_{r}}$ and $\bar{\alpha}_{m_{r}}$ denotes the conjugate of $\alpha_{m_{r}}$.

Our work involves a number of generalizations and extensions of known results that may be of some independent interest. For example, our results allow us to deduce the following theorem (see the remark at the end of Section 5).

Theorem 1.5. For $r \geq 3$, let $L$ denote the minimum period length of the continued fraction expansion for $\alpha_{m_{r}}$. Then the convergent $p_{t} / q_{t}$ of $\alpha_{m_{r}}$ satisfies

$$
\frac{1}{\mu_{r} q_{t}^{2}}<\left|\alpha_{m_{r}}-\frac{p_{t}}{q_{t}}\right|
$$

if and only if $t$ is a positive integer satisfying $t \not \equiv-1 \bmod L$.

Thus, while it is well known that there are infinitely many solutions to

$$
\left|\alpha_{m_{r}}-\frac{p_{k}}{q_{k}}\right| \leq \frac{1}{\mu_{r} q_{k}^{2}},
$$

in view of Theorem 1.5 we now see that those solutions are precisely those $p_{k} / q_{k}$ for which the index $k$ is congruent to -1 modulo $L$.

Acknowledgments. Funding for this project was provided, in part, by a grant from the National Science Foundation.

2. Basic results involving continued fractions. Here we quickly review the classical theory of continued fractions (see, for example, [1], Modules 4 and 5). For a real number $\alpha$, we denote its (simple) continued fraction expansion by

$$
\alpha=a_{0}+\frac{1}{a_{1}+\frac{1}{a_{2}+\frac{1}{\ddots}}}=\left[a_{0}, a_{1}, a_{2}, \ldots\right] .
$$

We write

$$
\frac{p_{n}}{q_{n}}=\left[a_{0}, a_{1}, \ldots, a_{n}\right]
$$

for the $n$th convergent of $\alpha$ and $(\alpha)_{n}=\left[a_{n}, a_{n+1}, \ldots\right]$ for the $n$th complete quotient of $\alpha$. For $n>1, p_{n+1}=a_{n+1} p_{n}+p_{n-1}$ and $q_{n+1}=a_{n+1} q_{n}+q_{n-1}$. 
As

$$
\left|\alpha-\frac{p_{n}}{q_{n}}\right|=\frac{1}{q_{n}\left((\alpha)_{n+1} q_{n}+q_{n-1}\right)},
$$

it follows that

$$
\left|\alpha-\frac{p_{n}}{q_{n}}\right|=\frac{1}{A_{n} q_{n}^{2}}
$$

where

$$
A_{n}=\left[a_{n+1}, a_{n+2}, \ldots\right]+\left[0, a_{n}, a_{n-1}, \ldots, a_{1}\right] .
$$

Also we recall that

$$
\frac{p_{0}}{q_{0}}<\frac{p_{2}}{q_{2}}<\ldots<\alpha<\ldots<\frac{p_{3}}{q_{3}}<\frac{p_{1}}{q_{1}} .
$$

Two irrational numbers $\alpha$ and $\beta$ are equivalent, $\alpha \sim \beta$, if from some point onward their continued fraction expansions agree. Alternatively, $\alpha \sim \beta$ if and only if $\beta$ is a linear fractional transformation of $\alpha$, that is, if and only if there exist integers $A, B, C, D$ satisfying $A D-B C= \pm 1$ and

$$
\beta=\frac{A \alpha+B}{C \alpha+D} .
$$

The continued fraction for $\alpha$ is periodic if and only if $\alpha$ is a quadratic irrational. If $\alpha$ is a quadratic irrational, we denote its continued fraction expansion as

$$
\alpha=\left[a_{0}, a_{1}, \ldots, a_{n}, \overline{a_{n+1}, a_{n+2}, \ldots, a_{n+k}}\right],
$$

where $\left(a_{n+1}, a_{n+2}, \ldots, a_{n+k}\right)$ denotes a periodic string.

Finally, for a positive integer $t$, we introduce the notation

$$
\left[a_{0}, a_{1}, \ldots, a_{n},\left(a_{n+1}, a_{n+2}, \ldots, a_{n+k}\right)_{t}, \ldots\right]
$$

to indicate that the string $\left(a_{n+1}, a_{n+2}, \ldots, a_{n+k}\right)$ is repeated $t$ times.

3. Markoff forms and the Lagrange spectrum. In this section we provide an overview of the fundamental work of Markoff [6] (see [2, Chapter II] or [3, Chapter 2]). A positive integer $m$ is a Markoff number if there exist positive integers $r, s$, satisfying $r<s<m$ with $m, r$, and $s$ pairwise relatively prime, such that the triple $(m, r, s)$ is a solution to the diophantine equation

$$
x^{2}+y^{2}+z^{2}=3 x y z .
$$

We let $u$ denote the smallest positive integer satisfying $u \equiv \pm r s^{-1} \bmod m$. In view of the previous diophantine equation, there must exist a positive integer $v$ such that $u^{2}+1=v m$. Moreover, for $m \neq 1,2$, we have

$$
2 v<u<3 v \text { and } 2 u<m<3 u \text {. }
$$


For a Markoff number $m$, we define its associated Markoff form $f_{m}(x, y)$ by

$$
f_{m}(x, y)=m x^{2}+(3 m-2 u) x y+(v-3 u) y^{2} .
$$

By classical results it follows that

$\min \left\{\left|f_{m}(x, y)\right|:(x, y) \in \mathbb{Z}^{2},(x, y) \neq(0,0)\right\}=f_{m}(1,0)=f_{m}(u, m)=m$.

We write $\alpha_{m}$ for the positive root of $f_{m}(x, 1)=0$, that is,

$$
\alpha_{m}=\frac{2 u-3 m+\sqrt{9 m^{2}-4}}{2 m},
$$

and we remark that the conjugate of $\alpha_{m}, \bar{\alpha}_{m}$, is equivalent to $\alpha_{m}$, and satisfies $\bar{\alpha}_{m}<-1$, while $0<\alpha_{m}<1$. In order to clarify notation, we note that in this paper, $\alpha_{m}$ always represents the quantity defined in (3.2) while $(\alpha)_{n}$ denotes the $n$th complete quotient associated with a number $\alpha$.

Clearly we can write $f_{m}(x, y)=m\left(\alpha_{m} y-x\right)\left(\bar{\alpha}_{m} y-x\right)$. If $\alpha_{m} \sim \beta$, where

$$
\beta=\frac{D \alpha_{m}-B}{-C \alpha_{m}+A}
$$

$A D-B C=1$, then

$$
\begin{aligned}
f_{m}(A x+B y, C x+D y) \\
\quad=m\left(A-C \alpha_{m}\right)\left(A-C \bar{\alpha}_{m}\right)(\beta y-x)(\bar{\beta} y-x) .
\end{aligned}
$$

We order the Markoff numbers and denote them by $m_{1}<m_{2}<\ldots$ So, for example, 1, 2, 5, 13, 29 are the first five Markoff numbers. We write $u_{r}$ and $v_{r}$ for the integers $u$ and $v$ associated with the Markoff number $m_{r}$.

The basic structure of the continued fraction expansion for $\alpha_{m_{r}}$ is somewhat understood. In particular, $\alpha_{m_{1}}=[0, \overline{1}], \alpha_{m_{2}}=[0, \overline{2}]$, and for $r \geq 3$,

$$
\alpha_{m_{r}}=\left[0, \overline{d_{1}, d_{2}, \ldots, d_{L}}\right]=[0, \overline{2, W, 1,1,2}],
$$

where $L=L(r)$ is an even integer, and $W=W(r)$ is a (possibly empty) string of 1's and 2's and is a palindrome. Moreover, any run of 1's or 2's in the string $W$ is of even length. It will be convenient to write $\alpha_{m_{1}}=[0, \overline{1,1}]$, $\alpha_{m_{2}}=[0, \overline{2,2}]$; that is, we declare $L(1)=L(2)=2$. In all other cases, $L$ denotes the length of the smallest periodic string of partial quotients.

Given an irrational number $\alpha$, a fundamental question in diophantine approximation is to find the best possible constant $\mu(\alpha)$ such that there exist infinitely many positive integers $q$ satisfying

$$
q\|\alpha q\| \leq 1 / \mu(\alpha) .
$$

Thus

$$
\mu(\alpha)^{-1}=\liminf _{q \rightarrow \infty} q\|\alpha q\| .
$$

The collection of all such constants $\mu(\alpha)$ is referred to as the Lagrange spectrum. 
Markoff discovered that

$$
\mu\left(\alpha_{m_{r}}\right)=\sqrt{9 m_{r}^{2}-4} / m_{r},
$$

which we denote as $\mu_{r}$, and produced the following celebrated result.

TheOREM 3.1. Let $\alpha$ be an irrational number not equivalent to $\alpha_{m_{1}}$, $\alpha_{m_{2}}, \ldots, \alpha_{m_{r-1}}$. Then there are infinitely many positive integers $q$ satisfying

$$
q\|\alpha q\| \leq 1 / \mu_{r} .
$$

Furthermore, the constant $\mu_{r}$ is best possible for any $\alpha$ equivalent to $\alpha_{m_{r}}$.

Thus the Markoff constants, $\mu_{r}$, are the smallest elements in the Lagrange spectrum. It also immediately follows that the smallest accumulation point of the Lagrange spectrum is 3 .

4. Recurrence sequences and auxiliary quadratic forms. For a fixed, positive integer $r$, we define the recurrence sequence $\mathcal{Z}_{r}(n)$ by $\mathcal{Z}_{r}(0)=$ $0, \mathcal{Z}_{r}(1)=1$, and for $n>1$,

$$
\mathcal{Z}_{r}(n)=3 m_{r} \mathcal{Z}_{r}(n-1)-\mathcal{Z}_{r}(n-2) .
$$

We begin with two basic observations. The first identity immediately follows by induction and thus we suppress its proof.

LEMma 4.1. For positive integers $r$ and $n$,

$$
\mathcal{Z}_{r}(n)^{2}+\mathcal{Z}_{r}(n-1)^{2}-3 m_{r} \mathcal{Z}_{r}(n) \mathcal{Z}_{r}(n-1)=1 .
$$

Lemma 4.2. For a fixed positive integer $r, \mathcal{Z}_{r}(n-1) / \mathcal{Z}_{r}(n)$ is a strictly increasing function of $n$ and

$$
\lim _{n \rightarrow \infty} \frac{\mathcal{Z}_{r}(n-1)}{\mathcal{Z}_{r}(n)}=\frac{3 m_{r}-\sqrt{9 m_{r}^{2}-4}}{2} .
$$

If the integer $n \geq 1$ is viewed as fixed, then $\mathcal{Z}_{r}(n-1) / \mathcal{Z}_{r}(n)$ is a decreasing function of $r$. Finally, for any positive integers $r$ and $n$,

$$
\frac{\mathcal{Z}_{r}(n-1)}{\mathcal{Z}_{r}(n)}<\frac{3-\sqrt{5}}{2}
$$

Proof. We first fix $r$ and write $\mathcal{Z}(n)$ for $\mathcal{Z}_{r}(n)$. Two direct calculations reveal that $\mathcal{Z}(0) / \mathcal{Z}(1)=0$ and $\mathcal{Z}(1) / \mathcal{Z}(2)=1 /\left(3 m_{r}\right)$, and thus $\mathcal{Z}(0) / \mathcal{Z}(1)<\mathcal{Z}(1) / \mathcal{Z}(2)$. We now assume that for some $n$,

$$
\mathcal{Z}(n-1) / \mathcal{Z}(n)<\mathcal{Z}(n) / \mathcal{Z}(n+1),
$$

or equivalently,

$$
-\mathcal{Z}(n)^{2}<-\mathcal{Z}(n+1) \mathcal{Z}(n-1) .
$$

After adding $3 m_{r} \mathcal{Z}(n) \mathcal{Z}(n+1)$ to both sides of the previous inequality and recalling the recurrence $\mathcal{Z}(n+2)=3 m_{r} \mathcal{Z}(n+1)-\mathcal{Z}(n)$, we see that 
$\mathcal{Z}(n+2) \mathcal{Z}(n)<\mathcal{Z}(n+1)^{2}$, which implies

$$
\frac{\mathcal{Z}(n)}{\mathcal{Z}(n+1)}<\frac{\mathcal{Z}(n+1)}{\mathcal{Z}(n+2)}
$$

Thus $\mathcal{Z}_{r}(n-1) / \mathcal{Z}_{r}(n)$ is a strictly increasing function of $n$.

The characteristic polynomial associated with the recurrence for $\mathcal{Z}_{r}(n)$ is easily seen to be $X^{2}-3 m_{r} X+1$. Thus, after computing the smaller zero of this polynomial, it follows that

$$
\lim _{n \rightarrow \infty} \frac{\mathcal{Z}_{r}(n-1)}{\mathcal{Z}_{r}(n)}=\frac{3 m_{r}-\sqrt{9 m_{r}^{2}-4}}{2} .
$$

Adopting the previous induction argument and recalling the fact that the Markoff numbers $m_{r}$ are increasing, one can verify that for $s<r$, the inequality

$$
\frac{\mathcal{Z}_{r}(n-1)}{\mathcal{Z}_{r}(n)} \leq \frac{\mathcal{Z}_{s}(n-1)}{\mathcal{Z}_{s}(n)}
$$

holds for all $n \geq 1$. Thus for fixed $n, \mathcal{Z}_{r}(n-1) / \mathcal{Z}_{r}(n)$ is a decreasing function of $r$.

Combining the above observations and recalling that $m_{1}=1$, we conclude that

$$
\frac{\mathcal{Z}_{r}(n-1)}{\mathcal{Z}_{r}(n)} \leq \frac{\mathcal{Z}_{1}(n-1)}{\mathcal{Z}_{1}(n)}<\frac{3 m_{1}-\sqrt{9 m_{1}^{2}-4}}{2 m_{1}}=\frac{3-\sqrt{5}}{2},
$$

which completes the proof.

Let $C_{r}(n)$ be as defined in (1.3). Then Lemma 4.2 and identity (1.2) reveal

$$
\lim _{n \rightarrow \infty} C_{r}(n)=\lim _{n \rightarrow \infty} \frac{\sqrt{9 m_{r}^{2}-4}}{2 m_{r}}+\frac{3}{2}-\frac{3 m_{r}-\sqrt{9 m_{r}^{2}-4}}{2 m_{r}}=\mu_{r},
$$

as claimed in Section 1. Furthermore, the inequality of Lemma 4.2 immediately implies

$$
\frac{\sqrt{9 m_{r}^{2}-4}}{2 m_{r}}+\frac{3}{2}-\frac{\sqrt{5}-3}{2 m_{r}}<C_{r}(n) \leq \frac{\sqrt{9 m_{r}^{2}-4}}{2 m_{r}}+\frac{3}{2} .
$$

Thus we conclude that $\lim _{r \rightarrow \infty} C_{r}(n)=3$, which establishes Corollary 1.3.

We now explore several important connections between the recurrence sequence $\mathcal{Z}_{r}(n)$ and the numbers $\alpha_{m_{r}}$. We inspire these connections by first recalling the following well known result (see, for example, [3, pp. 23-24]).

LEMMA 4.3. If $\alpha_{m_{r}}=\left[0, \overline{d_{1}, \ldots, d_{L}}\right]$, then $p_{L-1}=u_{r}, q_{L-1}=m_{r}$, and $p_{L}=3 u_{r}-v_{r}$, and $q_{L}=3 m_{r}-u_{r}$.

Using the recurrence sequence $\mathcal{Z}_{r}(n)$, we now extend this classical result. 
THEOREM 4.4. If $\alpha_{m_{r}}=\left[0, \overline{d_{1}, \ldots, d_{L}}\right]$, then for $n \geq 1$,

$$
p_{n L-1}=u_{r} \mathcal{Z}_{r}(n)-\mathcal{Z}_{r}(n-1), \quad q_{n L-1}=m_{r} \mathcal{Z}_{r}(n) .
$$

Proof. Let $r \geq 1$ be fixed. We first claim that for all $n \geq 1$,

$$
\begin{aligned}
\frac{p_{n L-1}}{q_{n L-1}} & =\frac{p_{(n-1) L+(L-1)}}{q_{(n-1) L+(L-1)}}=\left[0,\left(d_{1}, \ldots, d_{L}\right)_{n-1}, d_{1}, \ldots, d_{L-1}\right] \\
& =\frac{u_{r} \mathcal{Z}_{r}(n)-\mathcal{Z}_{r}(n-1)}{m_{r} \mathcal{Z}_{r}(n)} .
\end{aligned}
$$

By the previous lemma we have

$$
\frac{p_{L-1}}{q_{L-1}}=\frac{u_{r}}{m_{r}} .
$$

As $\mathcal{Z}_{r}(1)=1$ and $\mathcal{Z}_{r}(0)=0$, it follows that

$$
\frac{u_{r} \mathcal{Z}_{r}(n)-\mathcal{Z}_{r}(n-1)}{m_{r} \mathcal{Z}_{r}(n)}=\frac{u_{r}}{m_{r}}
$$

and thus (4.4) holds for $n=1$.

Next we assume that for some $n$,

$$
\frac{p_{n L-1}}{q_{n L-1}}=\left[0,\left(d_{1}, \ldots, d_{L}\right)_{n-1}, d_{1}, \ldots, d_{L-1}\right]=\frac{u_{r} \mathcal{Z}_{r}(n)-\mathcal{Z}_{r}(n-1)}{m_{r} \mathcal{Z}_{r}(n)},
$$

and therefore

$$
\left[\left(d_{1}, \ldots, d_{L}\right)_{n-1}, d_{1}, \ldots, d_{L-1}\right]=\frac{m_{r} \mathcal{Z}_{r}(n)}{u_{r} \mathcal{Z}_{r}(n)-\mathcal{Z}_{r}(n-1)} .
$$

Hence we can write

$$
\begin{aligned}
\frac{p_{(n+1) L-1}}{q_{(n+1) L-1}} & =\left[0,\left(d_{1}, \ldots, d_{L}\right)_{n}, d_{1}, \ldots, d_{L-1}\right] \\
& =\left[0, d_{1}, \ldots, d_{L},\left(d_{1}, \ldots, d_{L}\right)_{n-1}, d_{1}, \ldots, d_{L-1}\right] \\
& =\left[0, d_{1}, d_{2}, \ldots, d_{L}, \frac{m_{r} \mathcal{Z}_{r}(n)}{u_{r} \mathcal{Z}_{r}(n)-\mathcal{Z}_{r}(n-1)}\right],
\end{aligned}
$$

which, together with a well known identity involving continued fractions, yields

$$
\frac{p_{(n+1) L-1}}{q_{(n+1) L-1}}=\frac{p_{L}\left(\frac{m_{r} \mathcal{Z}_{r}(n)}{u_{r} \mathcal{Z}_{r}(n)-\mathcal{Z}_{r}(n-1)}\right)+p_{L-1}}{q_{L}\left(\frac{m_{r} \mathcal{Z}_{r}(n)}{u_{r} \mathcal{Z}_{r}(n)-\mathcal{Z}_{r}(n-1)}\right)+q_{L-1}} .
$$

Therefore Lemma 4.3, together with the recurrence $\mathcal{Z}_{r}(n+1)=3 m_{r} \mathcal{Z}_{r}(n)-$ $\mathcal{Z}_{r}(n-1)$, and the identity $u_{r}^{2}+1=v_{r} m_{r}$ imply

$$
\begin{aligned}
\frac{p_{(n+1) L-1}}{q_{(n+1) L-1}} & =\frac{p_{L}\left(\frac{m_{r} \mathcal{Z}_{r}(n)}{u_{r} \mathcal{Z}_{r}(n)-\mathcal{Z}_{r}(n-1)}\right)+p_{L-1}}{q_{L}\left(\frac{m_{r} \mathcal{Z}_{r}(n)}{u_{r} \mathcal{Z}_{r}(n)-\mathcal{Z}_{r}(n-1)}\right)+q_{L-1}} \\
& =\frac{\left(3 u_{r}-v_{r}\right)\left(\frac{m_{r} \mathcal{Z}_{r}(n)}{u_{r} \mathcal{Z}_{r}(n)-\mathcal{Z}_{r}(n-1)}\right)+u_{r}}{\left(3 m_{r}-u_{r}\right)\left(\frac{m_{r} \mathcal{Z}_{r}(n)}{u_{r} \mathcal{Z}_{r}(n)-\mathcal{Z}_{r}(n-1)}\right)+m_{r}}
\end{aligned}
$$




$$
\begin{aligned}
& =\frac{3 u_{r} m_{r} \mathcal{Z}_{r}(n)-v_{r} m_{r} \mathcal{Z}_{r}(n)+u_{r}^{2} \mathcal{Z}_{r}(n)-u_{r} \mathcal{Z}_{r}(n-1)}{3 m_{r}^{2} \mathcal{Z}_{r}(n)-u_{r} m_{r} \mathcal{Z}_{r}(n)+m_{r} u_{r} \mathcal{Z}_{r}(n)-m_{r} \mathcal{Z}_{r}(n-1)} \\
& =\frac{3 u_{r} m_{r} \mathcal{Z}_{r}(n)-\mathcal{Z}_{r}(n)-u_{r} \mathcal{Z}_{r}(n-1)}{3 m_{r}^{2} \mathcal{Z}_{r}(n)-m_{r} \mathcal{Z}_{r}(n-1)} \\
& =\frac{u_{r}\left(3 m_{r} \mathcal{Z}_{r}(n)-\mathcal{Z}_{r}(n-1)\right)-\mathcal{Z}_{r}(n)}{m_{r}\left(3 m_{r} \mathcal{Z}_{r}(n)-\mathcal{Z}_{r}(n-1)\right)} \\
& =\frac{u_{r} \mathcal{Z}_{r}(n+1)-\mathcal{Z}_{r}(n)}{m_{r} \mathcal{Z}_{r}(n+1)}
\end{aligned}
$$

which establishes our claim.

Finally, we demonstrate that $u_{r} \mathcal{Z}_{r}(n)-\mathcal{Z}_{r}(n-1)$ and $m_{r} \mathcal{Z}_{r}(n)$ are relatively prime. To establish this assertion, we apply the identity $u_{r}^{2}+1=$ $v_{r} m_{r}$ and Lemma 4.1 to deduce

$$
\begin{aligned}
\left(v_{r} \mathcal{Z}_{r}(n)-3 \mathcal{Z}_{r}(n-1)\right)\left(m_{r} \mathcal{Z}_{r}(n)\right) & \quad-\left(u_{r} \mathcal{Z}_{r}(n)+\mathcal{Z}_{r}(n-1)\right)\left(u_{r} \mathcal{Z}_{r}(n)-\mathcal{Z}_{r}(n-1)\right) \\
= & m_{r} v_{r} \mathcal{Z}_{r}(n)^{2}-3 m_{r} \mathcal{Z}_{r}(n) \mathcal{Z}_{r}(n-1) \\
& \quad-\left(u_{r}^{2} \mathcal{Z}_{r}(n)^{2}+u_{r} \mathcal{Z}_{r}(n) \mathcal{Z}_{r}(n-1)-u_{r} \mathcal{Z}_{r}(n) \mathcal{Z}_{r}(n-1)-\mathcal{Z}_{r}(n-1)^{2}\right) \\
= & \left(m_{r} v_{r}-u_{r}^{2}\right) \mathcal{Z}_{r}(n)^{2}+\mathcal{Z}_{r}(n-1)^{2}-3 m_{r} \mathcal{Z}_{r}(n) \mathcal{Z}_{r}(n-1) \\
= & \mathcal{Z}_{r}(n)^{2}+\mathcal{Z}_{r}(n-1)^{2}-3 m_{r} \mathcal{Z}_{r}(n) \mathcal{Z}_{r}(n-1)=1 .
\end{aligned}
$$

Thus we have

$$
\begin{aligned}
& \operatorname{gcd}\left(u_{r} \mathcal{Z}_{r}(n)-\mathcal{Z}_{r}(n-1), m_{r} \mathcal{Z}_{r}(n)\right) \\
& \quad=\operatorname{gcd}\left(v_{r} \mathcal{Z}_{r}(n)-3 \mathcal{Z}_{r}(n-1), u_{r} \mathcal{Z}_{r}(n)+\mathcal{Z}_{r}(n-1)\right)=1,
\end{aligned}
$$

which, in view of our claim, completes the proof.

The previous theorem and the definition of $\alpha_{m_{r}}$ given in (3.2), imply

$$
\begin{aligned}
-\bar{\alpha}_{m_{r}} & +\frac{p_{n L-1}}{q_{n L-1}} \\
= & -\frac{2 u_{r}-3 m_{r}-\sqrt{9 m_{r}^{2}-4}}{2 m_{r}}+\frac{u_{r} \mathcal{Z}_{r}(n)-\mathcal{Z}_{r}(n-1)}{m_{r} \mathcal{Z}_{r}(n)} \\
& =\frac{\sqrt{9 m_{r}^{2}-4}}{2 m_{r}}+\frac{2 u_{r} \mathcal{Z}_{r}(n)-2 \mathcal{Z}_{r}(n-1)-2 u_{r} \mathcal{Z}_{r}(n)+3 m_{r} \mathcal{Z}_{r}(n)}{2 m_{r} \mathcal{Z}_{r}(n)} \\
& =\frac{\sqrt{9 m_{r}^{2}-4}}{2 m_{r}}+\frac{3}{2}-\frac{\mathcal{Z}_{r}(n-1)}{m_{r} \mathcal{Z}_{r}(n)} \\
& =C_{r}(n) .
\end{aligned}
$$

Thus as an immediate consequence of Theorem 4.4 we have the following. 
Corollary 4.5. Let $p_{l} / q_{l}$ denote the lth convergent of $\alpha_{m_{r}}$. Then

$$
C_{r}(n)=-\bar{\alpha}_{m_{r}}+\frac{p_{n L-1}}{q_{n L-1}} .
$$

An adaptation of the argument used in Theorem 4.4 together with identity (4.2) allow us to deduce the following result which we state without proof.

TheOREM 4.6. Given the notation as in Theorem 4.4, for $r \geq 3$ and $n \geq 1$,

$$
p_{n L-3}=v_{r} \mathcal{Z}_{r}(n)-3 \mathcal{Z}_{r}(n-1), \quad q_{n L-3}=u_{r} \mathcal{Z}_{r}(n)+\mathcal{Z}_{r}(n-1) .
$$

\section{Moreover}

$$
q_{n L-3} p_{n L-1}-p_{n L-3} q_{n L-1}=-1 .
$$

We now define a sequence of auxiliary quadratic irrational numbers $\lambda_{r}(n)$ that allow us to better understand the diophantine nature of $\alpha_{m_{r}}$. Specifically, we exploit a connection between these auxiliary numbers and the convergents of $\alpha_{m_{r}}$. For integers $r \geq 3$ and $n \geq 1$, we define

$$
\lambda_{r}(n)=\frac{p_{n L-3}-p_{n L-1} \alpha_{m_{r}}}{q_{n L-3}-q_{n L-1} \alpha_{m_{r}}}
$$

where, again, $p_{l} / q_{l}$ denotes the $l$ th convergent associated with $\alpha_{m_{r}}$. We note that in view of identity (4.7), $\lambda_{r}(n)$ is equivalent to $\alpha_{m_{r}}$.

Lemma 4.7. Let $r \geq 3$ be a fixed integer. Then $\lambda_{r}(1)=3+\alpha_{m_{r}}$, and for $n \geq 2, \lambda_{r}(n)$ satisfies the recurrence relation

$$
\lambda_{r}(n)=\frac{u_{r} \lambda_{r}(n-1)+\left(3 u_{r}-v_{r}\right)}{m_{r} \lambda_{r}(n-1)+\left(3 m_{r}-u_{r}\right)} .
$$

Proof. By Lemma 4.3, we have $p_{L-1} / q_{L-1}=u_{r} / m_{r}$ and by a similar classical result, $p_{L-3} / q_{L-3}=v_{r} / u_{r}$. Thus,

$$
\lambda_{r}(1)=\frac{v_{r}-u_{r} \alpha_{m_{r}}}{u_{r}-m_{r} \alpha_{m_{r}}}
$$

which, in view of (3.2) and $v_{r} m_{r}-u_{r}^{2}=1$, reveals $\lambda_{r}(1)=3+\alpha_{m_{r}}$.

Next, substituting identity (4.8) into the expression

$$
\frac{u_{r} \lambda_{r}(n)+\left(3 u_{r}-v_{r}\right)}{m_{r} \lambda_{r}(n)+\left(3 m_{r}-u_{r}\right)}
$$

and applying Theorems 4.4 and 4.6, we conclude that

$$
\begin{aligned}
& \frac{u_{r} \lambda_{r}(n)+\left(3 u_{r}-v_{r}\right)}{m_{r} \lambda_{r}(n)+\left(3 m_{r}-u_{r}\right)} \\
= & \frac{\left(3 u_{r}-v_{r}\right) q_{n L-3}+u_{r} p_{n L-3}-\left(\left(3 u_{r}-v_{r}\right) q_{n L-1}+u_{r} p_{n L-1}\right) \alpha_{m_{r}}}{\left(3 m_{r}-u_{r}\right) q_{n L-3}+m_{r} p_{n L-3}-\left(\left(3 m_{r}-u_{r}\right) q_{n L-1}+u_{r} p_{n L-1}\right) \alpha_{m_{r}}} .
\end{aligned}
$$


In view of Theorems 4.4 and 4.6 , the identity $u_{r}^{2}+1=v_{r} m_{r}$, and the recursion relation for $\mathcal{Z}_{r}(n)$, we see

$$
\begin{aligned}
\left(3 u_{r}-v_{r}\right) q_{n L-1}+ & u_{r} p_{n L-1} \\
& =\left(3 u_{r}-v_{r}\right) m_{r} \mathcal{Z}_{r}(n)+u_{r}\left(u_{r} \mathcal{Z}_{r}(n)-\mathcal{Z}_{r}(n-1)\right) \\
& =u_{r}\left(3 m_{r} \mathcal{Z}_{r}(n)-\mathcal{Z}_{r}(n-1)\right)+\left(u_{r}^{2}-m_{r} v_{r}\right) \mathcal{Z}_{r}(n) \\
& =u_{r} \mathcal{Z}_{r}(n+1)-\mathcal{Z}_{r}(n) \\
& =p_{(n+1) L-1} .
\end{aligned}
$$

Similar arguments reveal that

$$
\begin{aligned}
\left(3 u_{r}-v_{r}\right) q_{n L-3}+u_{r} p_{n L-3} & =p_{(n+1) L-3}, \\
\left(3 m_{r}-u_{r}\right) q_{n L-1}+m_{r} p_{n L-1} & =q_{(n+1) L-1}, \\
\left(3 m_{r}-u_{r}\right) q_{n L-3}+m_{r} p_{n L-3} & =q_{(n+1) L-3} .
\end{aligned}
$$

These four identities, together with (4.9), imply

$$
\frac{u_{r} \lambda_{r}(n)+\left(3 u_{r}-v_{r}\right)}{m_{r} \lambda_{r}(n)+\left(3 m_{r}-u_{r}\right)}=\frac{p_{(n+1) L-3}-p_{(n+1) L-1} \alpha_{m_{r}}}{q_{(n+1) L-3}-q_{(n+1) L-1} \alpha_{m_{r}}}=\lambda_{r}(n+1),
$$

which completes the proof.

LEMMA 4.8. For integers $r \geq 3$ and $n \geq 1$,

$$
\left|\lambda_{r}(n)-\overline{\lambda_{r}(n)}\right|=\frac{\sqrt{9 m_{r}^{2}-4}}{m_{r}\left(q_{n L-3}-q_{n L-1} \alpha_{m_{r}}\right)\left(q_{n L-3}-q_{n L-1} \bar{\alpha}_{m_{r}}\right)},
$$

where $p_{l} / q_{l}$ denotes the lth convergent of $\alpha_{m_{r}}$.

The lemma follows easily from the definition of $\lambda_{r}(n)$ together with Theorem 4.6 and the observation that

$$
\alpha_{m_{r}}-\bar{\alpha}_{m_{r}}=\sqrt{9 m_{r}^{2}-4} / m_{r} .
$$

We now produce a critical result that connects the auxiliary numbers $\lambda_{r}(n)$ with the $\alpha_{m_{r}}$ and a subsequence of their convergents. We begin with the following lemma which is a special case of a more general phenomenon; however for ease of exposition we consider it separately.

LEMMA 4.9. For $r \geq 3$, the following string of inequalities holds

$$
\alpha_{m_{r}}<\frac{p_{L-1}}{q_{L-1}}<\overline{\lambda_{r}(1)}<\frac{p_{L-3}}{q_{L-3}}<\lambda_{r}(1),
$$

where $p_{l} / q_{l}$ denotes the lth convergent associated with $\alpha_{m_{r}}$.

Proof. As $r$ is fixed, we suppress the subscript $r$. From Lemma 4.3 we have $p_{L-1} / q_{L-1}=u / m$ and $p_{L-3} / q_{L-3}=v / u$. By Lemma 4.7 we have $\lambda(1)=3+\alpha_{m}$. As $\alpha_{m}=[0, \overline{2, W, 1,1,2}]$, where $W$ is some finite string, 
it follows that $p_{1} / q_{1}=1 / 2$. Finally we recall that $L$ is even and the oddindexed convergents form a decreasing sequence. Given these remarks, it is sufficient to prove

$$
\alpha_{m}<\frac{u}{m}<3+\bar{\alpha}_{m}<\frac{v}{u} \leq \frac{1}{2}<3+\alpha_{m} .
$$

As $p_{L-1} / q_{L-1}=u / m$ and $L-1$ is odd, we have that $\alpha_{m}<u / m$. Given identity (3.2), a direct calculation reveals that

$$
3+\bar{\alpha}_{m}-\frac{u}{m}=\frac{3 m-\sqrt{9 m^{2}-4}}{2 m}>0,
$$

thus, $u / m<3+\bar{\alpha}_{m}$. A slightly more elaborate calculation involving the identity $u^{2}+1=v m$, yields

$$
\frac{v}{u}-\left(3+\bar{\alpha}_{m}\right)=\frac{1}{m u}+\frac{\sqrt{9 m^{2}-4}-3 m}{2 m} .
$$

It follows that $3+\bar{\alpha}_{m}<v / u$ if and only if $u\left(3 m-\sqrt{9 m^{2}-4}\right)<2$. From (3.1), we have $2 u<m$, and hence

$$
u\left(3 m-\sqrt{9 m^{2}-4}\right)<\frac{1}{2} m\left(3 m-\sqrt{9 m^{2}-4}\right) .
$$

It is easily verified that the function $f(x)=\frac{1}{2} x\left(3 x-\sqrt{9 x^{2}-4}\right)$ is decreasing for $x \geq 1$. Thus for $x \geq 1, f(x) \leq f(1)=\frac{1}{2}(3-\sqrt{5})<2$, and therefore $u\left(3 m-\sqrt{9 m^{2}-4}\right)<2$, which implies that $3+\bar{\alpha}_{m}<v / u$.

Clearly

$$
\frac{v}{u}=\frac{p_{L-3}}{q_{L-3}} \leq \frac{p_{1}}{q_{1}}=\frac{1}{2}
$$

and $1 / 2<3+\alpha_{m}$, which completes the proof.

We now produce an important generalization of the previous lemma.

THEOREM 4.10. For $r \geq 3, n \geq 1$, and $k$ an odd integer satisfying $3 \leq$ $k \leq L-1$, the following string of inequalities holds:

$$
\alpha_{m_{r}}<\frac{p_{n L-1}}{q_{n L-1}}<\overline{\lambda_{r}(n)}<\frac{p_{n L-k}}{q_{n L-k}}<\lambda_{r}(n)
$$

where $p_{l} / q_{l}$ denotes the lth convergent associated with $\alpha_{m_{r}}$.

Proof. As the index $n L-1$ is odd, we immediately see that $\alpha_{m_{r}}<$ $p_{n L-1} / q_{n L-1}$. For the remaining string of inequalities, we proceed by induction on $n$.

The case $n=1$ follows from Lemma 4.9. Suppose now that the inequalities of (4.10) hold for $n$. For any $k, 3 \leq k \leq L-1$, we observe that

$$
\begin{aligned}
\frac{p_{(n+1) L-k}}{q_{(n+1) L-k}} & =\left[0,\left(d_{1}, d_{2}, \ldots, d_{L}\right)_{n}, d_{1}, d_{2}, \ldots, d_{L-k}\right] \\
& =\left[0, d_{1}, d_{2}, \ldots, d_{L},\left(d_{1}, d_{2}, \ldots, d_{L}\right)_{n-1}, d_{1}, d_{2}, \ldots, d_{L-k}\right] .
\end{aligned}
$$


By a well known identity involving continued fractions and Lemma 4.3, we see that

$$
\frac{p_{(n+1) L-k}}{q_{(n+1) L-k}}=\frac{p_{L} \frac{1}{\frac{p_{n L-k}}{q_{n L-k}}}+p_{L-1}}{q_{L} \frac{1}{\frac{p_{n L-k}}{q_{n L-k}}}+q_{L-1}}=\frac{u_{r}\left(\frac{p_{n L-k}}{q_{n L-k}}\right)+\left(3 u_{r}-v_{r}\right)}{m_{r}\left(\frac{p_{n L-k}}{q_{n L-k}}\right)+\left(3 m_{r}-u_{r}\right)} .
$$

Using the identity $u_{r}^{2}+1=v_{r} m_{r}$, one can verify that the function $F$ : $(0,+\infty) \rightarrow(0,+\infty)$ defined by

$$
F(x)=\frac{u_{r} x+\left(3 u_{r}-v_{r}\right)}{m_{r} x+\left(3 m_{r}-u_{r}\right)},
$$

is strictly increasing.

Thus, in view of our inductive hypothesis (4.10), we have

$$
F\left(\frac{p_{n L-1}}{q_{n L-1}}\right)<F\left(\overline{\lambda_{r}(n)}\right)<F\left(\frac{p_{n L-k}}{q_{n L-k}}\right)<F\left(\lambda_{r}(n)\right) .
$$

The previous string of inequalities together with (4.11) and Lemma 4.7 reveal

$$
\frac{p_{(n+1) L-1}}{q_{(n+1) L-1}}<\overline{\lambda_{r}(n+1)}<\frac{p_{(n+1) L-k}}{q_{(n+1) L-k}}<\lambda_{r}(n+1),
$$

which completes the proof.

Finally we will utilize the following result which connects the auxiliary numbers $\lambda_{r}(n)$ with the Markoff forms. For a fixed $r$, we define the quadratic form $h_{n}(x, y)$ by

$$
h_{n}(x, y)=h_{n}(r ; x, y)=m_{r}\left(\lambda_{r}(n) y-x\right)\left(\overline{\lambda_{r}(n)} y-x\right) \in \mathbb{Z}[x, y] .
$$

Lemma 4.11. For $r \geq 3$ and $n \geq 1$,

$$
\begin{aligned}
& f_{m_{r}}\left(q_{n L-3} x-p_{n L-3} y, q_{n L-1} x-p_{n L-1} y\right) \\
& \quad=\left(q_{n L-3}-q_{n L-1} \alpha_{m_{r}}\right)\left(q_{n L-3}-q_{n L-1} \bar{\alpha}_{m_{r}}\right) h_{n}(x, y) .
\end{aligned}
$$

Proof. In view of $(4.7)$, we have $\left(q_{n L-3}\right)\left(-p_{n L-1}\right)-\left(-p_{n L-3}\right)\left(q_{n L-1}\right)=$ 1. Thus the lemma follows from the definition of $\lambda_{r}(n)$ given in (4.8) and identity (3.3).

5. The proof of Theorem 1.2 in the case $\alpha=\alpha_{m_{r}}$. In this section we consider our main result in the case when $\alpha=\alpha_{m_{r}}$ and demonstrate that there are exactly $n$ integer solutions to the inequality appearing in Theorem 1.2 with equality holding for one of the $n$ solutions. Specifically we prove the following.

TheORem 5.1. Let $r$ be a positive integer. For $r=1$ and $r=2$, let $L=2$, and for $r \geq 3$, let $L$ denote the smallest period length of the continued 
fraction expansion for $\alpha_{m_{r}}$. Then for any positive integer $n$, the inequality

$$
\left|\alpha_{m_{r}}-\frac{p}{q}\right| \leq \frac{1}{C_{r}(n) q^{2}}
$$

has exactly $n$ rational solutions $p / q$. In particular, the only rational numbers $p / q$ satisfying inequality (5.1) are

$$
\frac{p_{L-1}}{q_{L-1}}, \frac{p_{2 L-1}}{q_{2 L-1}}, \ldots, \frac{p_{n L-1}}{q_{n L-1}},
$$

where $p_{l} / q_{l}$ denotes the lth convergent of $\alpha_{m_{r}}$. Moreover,

$$
\left|\alpha_{m_{r}}-\frac{p_{n L-1}}{q_{n L-1}}\right|=\frac{1}{C_{r}(n) q_{n L-1}^{2}} .
$$

We begin by verifying identity (5.2).

Lemma 5.2. If $\alpha_{m_{r}}=\left[0, \overline{d_{1}, \ldots, d_{L}}\right]$, then for $n \geq 1$,

$$
\left|\alpha_{m_{r}}-\frac{p_{n L-1}}{q_{n L-1}}\right|=\frac{1}{C_{r}(n) q_{n L-1}^{2}} .
$$

Proof. As $r$ is fixed, we suppress the subscript $r$. We begin by recalling that

and

$$
\alpha_{m}=\frac{2 u-3 m+\sqrt{9 m^{2}-4}}{2 m},
$$

$$
C(n)=\frac{\sqrt{9 m^{2}-4}}{2 m}+\frac{3}{2}-\frac{\mathcal{Z}(n-1)}{m \mathcal{Z}(n)} .
$$

We also recall our convention that for $\alpha_{m_{1}}=[0, \overline{1,1}]$ and $\alpha_{m_{2}}=[0, \overline{2,2}]$, we declare $L=2$. Thus for any $\alpha_{m}, L$ is known to be even, and thus $n L-1$ is odd. This simple observation implies that $\alpha_{m}<p_{n L-1} / q_{n L-1}$. Hence Lemma 4.4 reveals

$$
\begin{aligned}
C(n) \mid \alpha_{m} & -\frac{p_{n L-1}}{q_{n L-1}} \mid \\
= & \left(\frac{\sqrt{9 m^{2}-4}}{2 m}+\frac{3}{2}-\frac{\mathcal{Z}(n-1)}{m \mathcal{Z}(n)}\right)\left(\frac{p_{n L-1}}{q_{n L-1}}-\frac{2 u-3 m+\sqrt{9 m^{2}-4}}{2 m}\right) \\
= & \left(\frac{\sqrt{9 m^{2}-4}}{2 m}+\frac{3}{2}-\frac{\mathcal{Z}(n-1)}{m \mathcal{Z}(n)}\right) \\
& \times\left(\frac{u \mathcal{Z}(n)-\mathcal{Z}(n-1)}{m \mathcal{Z}(n)}-\frac{2 u-3 m+\sqrt{9 m^{2}-4}}{2 m}\right) \\
= & \left(\frac{3}{2}-\frac{\mathcal{Z}(n-1)}{m \mathcal{Z}(n)}+\frac{\sqrt{9 m^{2}-4}}{2 m}\right)\left(\frac{3}{2}-\frac{\mathcal{Z}(n-1)}{m \mathcal{Z}(n)}-\frac{\sqrt{9 m^{2}-4}}{2 m}\right) \\
= & \frac{\mathcal{Z}(n)^{2}+\mathcal{Z}(n-1)^{2}-3 m \mathcal{Z}(n) \mathcal{Z}(n-1)}{(m \mathcal{Z}(n))^{2}} .
\end{aligned}
$$


In view of identity (4.2), and the fact that $q_{n L-1}=m \mathcal{Z}(n)$ from Theorem 4.4 , we see that

$$
\left|\alpha_{m}-\frac{p_{n L-1}}{q_{n L-1}}\right| C(n)=\frac{1}{q_{n L-1}^{2}},
$$

which establishes our desired identity.

Next we show that the convergents in Theorem 5.1 satisfy inequality (5.1).

LEMma 5.3. For $n \geq 1$, the $n$ rational numbers

$$
\frac{p_{L-1}}{q_{L-1}}, \frac{p_{2 L-1}}{q_{2 L-1}}, \ldots, \frac{p_{n L-1}}{q_{n L-1}}
$$

each satisfy the inequality

$$
\left|\alpha_{m_{r}}-\frac{p}{q}\right| \leq \frac{1}{C_{r}(n) q^{2}} .
$$

Proof. Again, as $r$ is fixed, we suppress the subscript $r$. By the previous lemma, for any integer $l$ we have

$$
\left|\alpha_{m}-\frac{p_{l L-1}}{q_{l L-1}}\right|=\frac{1}{C(l) q_{l L-1}^{2}} .
$$

By Lemma 4.2 , the ratio $\mathcal{Z}(n-1) / \mathcal{Z}(n)$ is increasing as a function of $n$. Thus, as the Markoff number $m$ is fixed, it follows that $C(n)$ is a decreasing function of $n$. Therefore for any $l, 1 \leq l \leq n$,

$$
\left|\alpha_{m}-\frac{p_{l L-1}}{q_{l L-1}}\right|=\frac{1}{C(l) q_{l L-1}^{2}} \leq \frac{1}{C(n) q_{l L-1}^{2}},
$$

which completes the proof.

Finally we demonstrate that there are no other rational solutions to (5.1), that is, the $n$ convergents in Lemma 5.3 form the complete list of rational solutions. We begin by considering convergents $p / q$ for which $p / q<$ $p_{n L-1} / q_{n L-1}$.

Lemma 5.4. Let $r$ and $n$ be positive integers. If $p / q$ is a convergent of $\alpha_{m_{r}}$ satisfying $p / q<p_{n L-1} / q_{n L-1}$, then

$$
\frac{1}{C_{r}(n) q^{2}}<\left|\alpha_{m_{r}}-\frac{p}{q}\right| .
$$

Proof. In Section 3 we noted that

$$
\min \left\{\left|f_{m_{r}}(x, y)\right|:(x, y) \in \mathbb{Z}^{2},(x, y) \neq(0,0)\right\}=m_{r} .
$$

Thus we see that

$$
\begin{aligned}
\frac{m_{r}}{q^{2}} & \leq \frac{\left|f_{m_{r}}(p, q)\right|}{q^{2}}=\frac{\left|m_{r}\left(\alpha_{m_{r}} q-p\right)\left(\bar{\alpha}_{m_{r}} q-p\right)\right|}{q^{2}} \\
& =m_{r}\left|\alpha_{m_{r}}-\frac{p}{q}\right| \cdot\left|\bar{\alpha}_{m_{r}}-\frac{p}{q}\right| .
\end{aligned}
$$


From (3.1) and (3.2), it follows that $\bar{\alpha}_{m_{r}}<0$. Since

$$
\bar{\alpha}_{m_{r}}<0 \leq \frac{p}{q}<\frac{p_{n L-1}}{q_{n L-1}},
$$

by Corollary 4.5 we have

$$
\left|\bar{\alpha}_{m_{r}}-\frac{p}{q}\right|<\left|\bar{\alpha}_{m_{r}}-\frac{p_{n L-1}}{q_{n L-1}}\right|=C_{r}(n) .
$$

The previous inequality together with (5.4) reveal

$$
\frac{m_{r}}{q^{2}} \leq m_{r}\left|\alpha_{m_{r}}-\frac{p}{q}\right| \cdot\left|\bar{\alpha}_{m_{r}}-\frac{p}{q}\right|<m_{r}\left|\alpha_{m_{r}}-\frac{p}{q}\right| C_{r}(n),
$$

which establishes the lemma.

We now consider convergents $p / q$ satisfying $p / q>p_{n L-1} / q_{n L-1}$. It is enough to consider those convergents of the form $p_{l L-k} / q_{l L-k}$, where $k$ is an odd integer satisfying $3 \leq k \leq L-1$.

Theorem 5.5. Let $r \geq 3$ and $l \geq 1$ be integers, and let $L$ be the period length of $\alpha_{m_{r}}$. For an odd integer $k, 3 \leq k \leq L-1$,

$$
\frac{1}{\mu_{r} q_{l L-k}^{2}}<\left|\alpha_{m_{r}}-\frac{p_{l L-k}}{q_{l L-k}}\right|
$$

where $\mu_{r}$ denotes the rth Markoff constant.

Proof. As $r$ is fixed, we again suppress the subscript $r$. The function

$$
h_{l}(x, y)=m(\lambda(l) y-x)(\overline{\lambda(l)} y-x),
$$

immediately yields

$$
\frac{\left|h_{l}\left(p_{l L-k}, q_{l L-k}\right)\right|}{q_{l L-k}^{2}}=m\left|\lambda(l)-\frac{p_{l L-k}}{q_{l L-k}}\right| \cdot\left|\overline{\lambda(l)}-\frac{p_{l L-k}}{q_{l L-k}}\right| .
$$

In view of Theorem 4.10, we are able to conclude

$$
\left|\overline{\lambda(l)}-\frac{p_{l L-k}}{q_{l L-k}}\right|<\left|\alpha_{m}-\frac{p_{l L-k}}{q_{l L-k}}\right| \text { and }\left|\lambda(l)-\frac{p_{l L-k}}{q_{l L-k}}\right|<|\lambda(l)-\overline{\lambda(l)}| \text {, }
$$

and by Lemma 4.8, the previous inequality can be written as

$$
\left|\lambda(l)-\frac{p_{l L-k}}{q_{l L-k}}\right|<\frac{\sqrt{9 m^{2}-4}}{m\left|\left(q_{l L-3}-q_{l L-1} \alpha_{m}\right)\left(q_{l L-3}-q_{l L-1} \bar{\alpha}_{m}\right)\right|} .
$$

The previous inequalities together with (5.6) reveal

$$
\begin{aligned}
& \frac{\left|h_{l}\left(p_{l L-k}, q_{l L-k}\right)\right|}{q_{l L-k}^{2}} \\
& \quad<\left|\alpha_{m}-\frac{p_{l L-k}}{q_{l L-k}}\right|\left(\frac{\sqrt{9 m^{2}-4}}{\left|\left(q_{l L-3}-q_{l L-1} \alpha_{m}\right)\left(q_{l L-3}-q_{l L-1} \bar{\alpha}_{m}\right)\right|}\right) .
\end{aligned}
$$


By Lemma 4.11 we have

$$
h_{l}(x, y)=\frac{f_{m}\left(q_{l L-3} x-p_{l L-3} y, q_{l L-1} x-p_{l L-1} y\right)}{\left(q_{l L-3}-q_{l L-1} \alpha_{m}\right)\left(q_{l L-3}-q_{l L-1} \bar{\alpha}_{m}\right)} .
$$

It is easily verified that $\left(q_{l L-3} p_{l L-k}-p_{l L-3} q_{l L-k}, q_{l L-1} p_{l L-k}-p_{l L-1} q_{l L-k}\right) \neq$ $(0,0)$, and thus as

$$
\min \left\{\left|f_{m}(x, y)\right|:(x, y) \in \mathbb{Z}^{2},(x, y) \neq(0,0)\right\}=m,
$$

inequality (5.7) implies

$$
\begin{aligned}
& \frac{m}{\left|\left(q_{l L-3}-q_{l L-1} \alpha_{m}\right)\left(q_{l L-3}-q_{l L-1} \bar{\alpha}_{m}\right)\right| q_{l L-k}^{2}} \\
& <\left|\alpha_{m}-\frac{p_{l L-k}}{q_{l L-k}}\right| \frac{\sqrt{9 m^{2}-4}}{\left|\left(q_{l L-3}-q_{l L-1} \alpha_{m}\right)\left(q_{l L-3}-q_{l L-1} \bar{\alpha}_{m}\right)\right|}
\end{aligned}
$$

and hence

$$
\frac{1}{q_{l L-k}^{2}}<\left|\alpha_{m}-\frac{p_{l L-k}}{q_{l L-k}}\right|\left(\frac{\sqrt{9 m^{2}-4}}{m}\right) .
$$

The theorem now follows from the fact that $\mu=\sqrt{9 m^{2}-4} / m$.

Proof of Theorem 5.1. By Lemma 5.3 we see that (5.1) holds for the $n$ convergents stated in the theorem, and Lemma 5.2 establishes identity (5.2). Thus we need only prove that there are no other rational solutions to inequality (5.1).

It is clear from the definition that $C_{r}(n)>2$. Thus if $p / q$ is not a convergent of $\alpha_{m_{r}}$, then by Legendre's Theorem,

$$
\left|\alpha_{m_{r}}-\frac{p}{q}\right| \geq \frac{1}{2 q^{2}}>\frac{1}{C_{r}(n) q^{2}} .
$$

Hence any rational $p / q$ satisfying (5.1) must be a convergent of $\alpha_{m_{r}}$.

By Lemma 5.4, no convergent $p / q<p_{n L-1} / q_{n L-1}$ can satisfy inequality (5.1). Thus we need only consider convergents $p / q$ that exceed $p_{n L-1} / q_{n L-1}$.

We again recall that for $r=1$ or $r=2$, we declare the period $L=2$. Thus, in either case, the only convergents exceeding $p_{n L-1} / q_{n L-1}$ are

$$
\frac{p_{1}}{q_{1}}, \frac{p_{3}}{q_{3}}, \ldots, \frac{p_{2 n-1}}{q_{2 n-1}}
$$

and therefore the theorem holds for $r=1$ and $r=2$ by Lemma 5.3.

We now assume that $r \geq 3$. If $p / q$ is a convergent exceeding $p_{n L-1} / q_{n L-1}$, then either there exists an integer $l, 1 \leq l \leq n$, such that

$$
\frac{p}{q}=\frac{p_{l L-1}}{q_{l L-1}},
$$


or there exist integers $l, 1 \leq l \leq n$, and $k, k$ odd with $3 \leq k \leq L-1$, such that

$$
\frac{p}{q}=\frac{p_{l L-k}}{q_{l L-k}} .
$$

As we have seen the convergents of the form (5.8) satisfy inequality (5.1), we now assume that (5.9) holds. By Theorem 5.5 we have

$$
\left|\alpha_{m_{r}}-\frac{p}{q}\right|=\left|\alpha_{m_{r}}-\frac{p_{l L-k}}{q_{l L-k}}\right|>\frac{1}{\mu_{r} q_{l L-k}^{2}} .
$$

By Lemma 4.2 and the remarks that followed it, we see that $C_{r}(n)>\mu_{r}$, and hence

$$
\left|\alpha_{m_{r}}-\frac{p_{l L-k}}{q_{l L-k}}\right|>\frac{1}{\mu_{r} q_{l L-k}^{2}}>\frac{1}{C_{r}(n) q_{l L-k}^{2}} .
$$

Thus the only rational solutions $p / q$ to inequality (5.1) are those $n$ rational numbers appearing in Lemma 5.3.

REMARK. As an aside, we claim that inequality (5.5) also holds for convergents $p_{t} / q_{t}$ with $t$ even. To establish this claim, if we assume that $t$ is even, then clearly $\bar{\alpha}_{m_{r}}<0<p_{t} / q_{t}<\alpha_{m_{r}}$. Hence

$$
\left|\bar{\alpha}_{m_{r}}-\frac{p_{t}}{q_{t}}\right|=\frac{p_{t}}{q_{t}}-\bar{\alpha}_{m_{r}}<\alpha_{m_{r}}-\bar{\alpha}_{m_{r}}=\frac{\sqrt{9 m_{r}^{2}-4}}{m_{r}}=\mu_{r} .
$$

Thus in view of inequality (5.4) we have

$$
\frac{1}{\mu_{r} q_{t}^{2}}<\left|\alpha_{m_{r}}-\frac{p_{t}}{q_{t}}\right|
$$

which verifies our claim and, together with Lemma 5.2, also establishes Theorem 1.5.

6. The proof of Theorem 1.2 when $\alpha$ is equivalent to $\alpha_{m_{r}}$. In this section we prove the following result.

THEOREM 6.1. Let $r$ and $n$ be positive integers and suppose that $\alpha$ is equivalent to $\alpha_{m_{r}}$ but $\alpha \neq \alpha_{m_{r}}$. Then there exist at least $n$ rational solutions to the inequality

$$
\left|\alpha-\frac{p}{q}\right| \leq \frac{1}{C_{r}(n) q^{2}} .
$$

The cases $r=1$ and $r=2$ have been studied in a number of papers. The following theorem summarizes the results of Prasad [7], Eggan [4], and Prasad and Prasad [8].

Theorem 6.2. Let $n$ be a positive integer, $r=1$ or 2 , and let $\alpha$ be equivalent to $\alpha_{m_{r}}$ but $\alpha \neq \alpha_{m_{r}}$. Then there exist at least $n$ rational solutions 
to the inequality

$$
\left|\alpha-\frac{p}{q}\right| \leq \frac{1}{C_{r}(n) q^{2}} .
$$

Thus for the remainder of this section, we assume $r \geq 3$ is fixed. We prove our theorem by exhibiting particular convergents of $\alpha$ satisfying inequality (6.1). If we write $\alpha=\left[a_{0}, a_{1}, \ldots\right]$, then we recall that any convergent $p_{l} / q_{l}$ of $\alpha$ satisfies

$$
\left|\alpha-\frac{p_{l}}{q_{l}}\right|=\frac{1}{A_{l} q_{l}^{2}}
$$

where

$$
A_{l}=\left[a_{l+1}, a_{l+2}, \ldots\right]+\left[0, a_{l}, a_{l-1}, \ldots, a_{1}\right] .
$$

Therefore, $p_{l} / q_{l}$ satisfies (6.1), if and only if $A_{l} \geq C_{r}(n)$. Thus it is enough to find $n$ convergents of $\alpha, p_{l} / q_{l}$, for which $A_{l} \geq C_{r}(n)$.

We begin with two technical lemmas. We write $\alpha_{m}$ for $\alpha_{m_{r}}$, and recall that $\alpha_{m}=[0, \overline{2, W, 1,1,2}]$, where $W$ is a finite string of 1 's and 2's forming a palindrome. We denote this expansion as $\alpha_{m}=\left[0, \overline{d_{1}, d_{2}, \ldots, d_{L}}\right]$. We now define

$$
\begin{aligned}
\delta_{m} & =2+\alpha_{m}=[2, \overline{2, W, 1,1,2}]=[\overline{2,2, W, 1,1}] \\
& =\left[\overline{d_{0}, d_{1}, \ldots, d_{L-1}}\right]
\end{aligned}
$$

and

$$
\gamma_{m}=[\overline{2,1,1, W, 2}]=\left[\overline{d_{L}, d_{L-1}, \ldots, d_{1}}\right] .
$$

We note that $\gamma_{m}$ is simply $1 / \alpha_{m}$ with its periodic string read backwards. We denote this observation by expressing the continued fraction expansion of $\gamma_{m}$ as

$$
\gamma_{m}=\left[\left[\frac{1}{\alpha_{m}}\right]_{\Leftarrow}\right] .
$$

LEMmA 6.3. The numbers $\delta_{m}$ and $\gamma_{m}$ are equivalent, and there exists an odd index $J, J<L$, such that the $J$ th complete quotient of $\delta_{m}$ equals $\gamma_{m}$. Moreover, for any $0 \leq i \leq L-1, d_{L-i}=d_{J+i}$.

Proof. Clearly $\delta_{m}$ is equivalent to $\alpha_{m}$. As we remarked in Section 3, $\alpha_{m} \sim \bar{\alpha}_{m}$, and thus $\alpha_{m} \sim-\bar{\alpha}_{m}$. By a well known identity involving purely periodic continued fractions (see, for instance, [1, Lemma 8.7]), we have

$$
\begin{aligned}
-\bar{\alpha}_{m} & =\frac{-1}{\overline{1 / \alpha_{m}}}=\left[\left[\frac{1}{\alpha_{m}}\right]_{\Leftarrow}\right]=\left[\left[\overline{d_{1}, d_{2}, \ldots, d_{L}}\right]_{\Leftarrow}\right] \\
& =\left[\overline{d_{L}, d_{L-1}, \ldots, d_{1}}\right]=\gamma_{m} .
\end{aligned}
$$

Since $\delta_{m} \sim \alpha_{m}$ and $\alpha_{m} \sim-\bar{\alpha}_{m}=\gamma_{m}$, we have $\delta_{m} \sim \gamma_{m}$. As $\gamma_{m}$ is purely periodic, there exists an index $J^{\prime}$ such that $\left(\delta_{m}\right)_{J^{\prime}}=\gamma_{m}$. Recalling that 
$\delta_{m}$ is purely periodic with period length $L$, it is clear that we may take $J=J^{\prime}-k L$ for some non-negative integer $k$ so that $\left(\delta_{m}\right)_{J}=\gamma_{m}$ and $J<L$. In view of (6.4) and (6.3), we now see

$$
\gamma_{m}=\left[\overline{d_{L}, d_{L-1}, \ldots, d_{2}, d_{1}}\right]=\left[\overline{d_{J}, d_{J+1}, \ldots, d_{J+(L-2)}, d_{J+(L-1)}}\right],
$$

and thus it is clear from our knowledge of the partial quotients of $\alpha_{m}$ as outlined in Section 3 that $d_{L-i}=d_{J+i}$ for all $i, 0 \leq i \leq L-1$. We also see that $d_{J}=d_{L}=2$ and $d_{L-1}=d_{J+1}=1$. As we know that each run of 1 's or 2's in the continued fraction of $\alpha_{m}$ is of even length, it follows that if $J$ were even, then we would have $d_{J}=d_{J+1}$, which is a contradiction. Thus we conclude that $J$ is odd, which establishes the lemma.

The following lemma connects $\delta_{m}$ and $\gamma_{m}$ with the constant $C_{r}(n)$.

LEMma 6.4. Given the notation as in Lemma 6.3,

$$
\begin{aligned}
C_{r}(n)= & \delta_{m}+\left[0,\left(d_{L-1}, d_{L-2}, \ldots, d_{0}\right)_{n-1}, d_{L-1}, d_{L-2}, \ldots, d_{1}\right] \\
= & \gamma_{m}+\left[0,\left(d_{J+(L-1)}, d_{J+(L-2)}, \ldots, d_{J}\right)_{n-1},\right. \\
& \left.d_{J+(L-1)}, d_{J+(L-2)}, \ldots, d_{J+1}\right] .
\end{aligned}
$$

Proof. We first note that

$$
\frac{p_{n L-1}}{q_{n L-1}}=\left[0,\left(d_{1}, d_{2}, \ldots, d_{L}\right)_{n-1}, d_{1}, d_{2}, \ldots, d_{L-1}\right],
$$

and by Theorem 5.2 ,

$$
\left|\alpha_{m}-\frac{p_{n L-1}}{q_{n L-1}}\right|=\frac{1}{C_{r}(n) q_{n L-1}^{2}} .
$$

From (6.2) it follows that

$$
C_{r}(n)=\left[d_{n L}, d_{n L+1}, \ldots\right]+\left[0, d_{n L-1}, d_{n L-2}, \ldots, d_{1}\right] .
$$

As the period length of $\alpha_{m}$ is $L$ and $d_{0}=d_{L}$, we can write

$$
\begin{aligned}
C_{r}(n) & =\left[d_{0}, d_{1}, \ldots\right]+\left[0, d_{L-1}, d_{L-2}, \ldots, d_{1},\left(d_{L}, d_{L-1}, \ldots, d_{1}\right)_{n-1}\right] \\
& =\left[\overline{d_{0}, d_{1}, \ldots, d_{L-1}}\right]+\left[0,\left(d_{L-1}, d_{L-2}, \ldots, d_{1}, d_{L}\right)_{n-1},\right. \\
& \left.=d_{L-1}, d_{L-2}, \ldots, d_{1}\right] \\
& \delta_{m}+\left[0,\left(d_{L-1}, d_{L-2}, \ldots, d_{1}, d_{0}\right)_{n-1}, d_{L-1}, d_{L-2}, \ldots, d_{1}\right] .
\end{aligned}
$$

Finally, by Corollary 4.5 we have

$$
C_{r}(n)=-\bar{\alpha}_{m}+\left[0, d_{1}, d_{2}, \ldots, d_{n L-1}\right] .
$$

In the proof of Lemma 6.3 we observed that $-\bar{\alpha}_{m}=\gamma_{m}$. Thus

$$
C_{r}(n)=\gamma_{m}+\left[0,\left(d_{1}, d_{2}, \ldots, d_{L}\right)_{n-1}, d_{1}, d_{2}, \ldots, d_{L-1}\right] .
$$

By Lemma $6.3, d_{J+i}=d_{L-i}$ for any non-negative $i$, so (6.5) yields 


$$
\begin{array}{r}
C_{r}(n)=\gamma_{m}+\left[0,\left(d_{J+(L-1)}, d_{J+(L-2)}, \ldots, d_{J}\right)_{n-1}, d_{J+(L-1)},\right. \\
\left.d_{J+(L-2)}, \ldots, d_{J+1}\right],
\end{array}
$$

which completes the proof.

We are now able to provide a proof of Theorem 6.1 for $r \geq 3$.

Proof of Theorem 6.1. By considering $\alpha-[\alpha]$ instead of $\alpha$, it follows that there is no loss of generality in assuming that $0<\alpha<1$. Let $r \geq 3$ be a fixed integer. For ease of notation, we again suppress the subscript $r$.

Our method of proof is to explicitly exhibit $n$ convergents of $\alpha$ that satisfy inequality (6.1). By assumption, $\alpha$ is equivalent to $\alpha_{m}$ and since $\alpha_{m} \sim \delta_{m}$, we have $\alpha \sim \delta_{m}$. We first consider the case when $\alpha$ is not equal to $1 / \delta_{m}=\left[0, \overline{d_{0}, d_{1}, \ldots, d_{L-1}}\right]$.

Since $\delta_{m}$ is purely periodic, there exists a smallest index, $T$, such that the $T$ th complete quotient $\alpha_{T}$ is $\delta_{m}$. Since $\alpha \neq 1 / \delta_{m}$, we have $T \geq 2$. Thus we can express the continued fraction of $\alpha$ as

$$
\alpha=\left[0, a_{1}, \ldots, a_{T-1}, a_{T}, \ldots\right]=\left[0, a_{1}, \ldots, a_{T-1}, \overline{d_{0}, d_{1}, \ldots, d_{L-1}}\right] .
$$

To make our exposition clearer, we re-index the partial quotients of $\alpha$ by defining $a_{L-1}^{\prime}=a_{T-1}, a_{L-2}^{\prime}=a_{T-2}$ and so forth with $a_{S}^{\prime}=a_{1}$, where $S$ is the appropriate integer (not necessarily positive). That is, we write

$$
\begin{aligned}
\alpha & =\left[0, a_{1}, a_{2}, \ldots, a_{T-2}, a_{T-1}, \overline{d_{0}, d_{1}, \ldots, d_{L-1}}\right] \\
& =\left[0, a_{S}^{\prime}, a_{S+1}^{\prime}, \ldots, a_{L-2}^{\prime}, a_{L-1}^{\prime}, \overline{d_{0}, d_{1}, \ldots, d_{L-1}}\right],
\end{aligned}
$$

and thus

$$
\left[a_{T}, a_{T+1}, \ldots\right]=\left[a_{L}^{\prime}, a_{L+1}^{\prime}, \ldots\right]=\delta_{m} .
$$

The advantage of this new notation is that it allows us to align indices and write

$$
a_{L+i}^{\prime}=d_{L+i}=d_{i}
$$

for all integers $i \geq 0$. Furthermore, as in (6.2), we define $A_{t}^{\prime}$ as

$$
A_{t}^{\prime}=\left[a_{t+1}^{\prime}, a_{t+2}^{\prime}, \ldots\right]+\left[0, a_{t}^{\prime}, a_{t-1}^{\prime}, \ldots, a_{S+1}^{\prime}, a_{S}^{\prime}\right]
$$

Of course for any given $A_{t}^{\prime}$ there exists a non-negative integer $u$ such that

$$
A_{t}^{\prime}=A_{u}=\left[a_{u+1}, a_{u+2}, \ldots\right]+\left[0, a_{u}, a_{u-1}, \ldots, a_{2}, a_{1}\right] .
$$

Thus to prove the theorem, it is enough to show that there exist $n$ indices, say $t_{1}, \ldots, t_{n}$, such that $A_{t_{i}}^{\prime} \geq C(n)$ for each $i=1, \ldots, n$.

For the remainder of the proof, references to the indices of partial quotients of $\alpha$ will refer to the indices of the $a^{\prime}$ terms. Of course each such index will have a corresponding index when the continued fraction expansion for $\alpha$ is expressed in terms of $a$ 's. We now consider two cases: 
(i) The index $S$ satisfies $S<0$.

(ii) The index $S$ satisfies $S \geq 0$.

We first examine case (i). Since the period length $L>0$ is the smallest index such that $\left[a_{L}^{\prime}, a_{L+1}^{\prime}, \ldots\right]=\delta_{m}$, we claim that there must exist an index $i, 0 \leq i \leq L-1$, such that $a_{i}^{\prime} \neq d_{i}$. If not, then we must have, $a_{i}^{\prime}=d_{i}$ for all $i \geq 0$. In this case, we would have $\left[a_{0}^{\prime}, a_{1}^{\prime}, \ldots\right]=\delta_{m}$, which contradicts the minimality of the index $L$ and therefore establishes our claim.

Thus there exists some index $i, 0 \leq i \leq L-1$, satisfying $a_{i}^{\prime} \neq d_{i}$. Let $k$ be the largest of all such indices. Therefore

$$
a_{i}^{\prime}=d_{i} \quad \text { for all } i, k+1 \leq i \leq L-1, \quad \text { and } \quad a_{k}^{\prime} \neq d_{k} .
$$

As $a_{k}^{\prime} \neq d_{k}$, it follows that one of the following two subcases must hold.

Subcase I. Either $a_{k}^{\prime}<d_{k}$ and $k$ is odd or $a_{k}^{\prime}>d_{k}$ and $k$ is even.

Subcase II. Either $a_{k}^{\prime}>d_{k}$ and $k$ is odd or $a_{k}^{\prime}<d_{k}$ and $k$ is even.

We now examine subcase I and note that either $k=0$ or $k \geq 1$. If $k=0$, then we have $a_{0}^{\prime}>d_{0}=2$ and thus $a_{0}^{\prime} \geq 3$. Moreover for all $i \geq 1, a_{i}^{\prime}=d_{i}$. Hence, in view of identity (6.8) and Lemma 4.2 we have

$$
A_{-1}^{\prime} \geq\left[a_{0}^{\prime}, a_{1}^{\prime}, \ldots\right] \geq\left[3, a_{1}^{\prime}, \ldots\right]>3>C(n) .
$$

More generally, for any $l, 2 \leq l \leq n$, we obtain

$$
\begin{aligned}
A_{(l-1) L-1}^{\prime}= & {\left[a_{(l-1) L}^{\prime}, a_{(l-1) L+1}^{\prime}, \ldots\right]+\left[0, a_{(l-1) L-1}^{\prime}, a_{(l-1) L-2}^{\prime}, \ldots, a_{S}^{\prime}\right] } \\
= & {\left[d_{(l-1) L}, d_{(l-1) L+1}, \ldots\right] } \\
& +\left[0, d_{(l-1) L-1}, d_{(l-1) L-2}, \ldots, d_{1}, a_{0}^{\prime}, \ldots, a_{S}^{\prime}\right] .
\end{aligned}
$$

As $d_{i}=d_{L+i}$, we may write the previous identity as

$$
\begin{aligned}
A_{(l-1) L-1}^{\prime}=\left[\overline{d_{0}, d_{1}, \ldots, d_{L-1}}\right]+ & {\left[0,\left(d_{L-1}, d_{L-2}, \ldots, d_{0}\right)_{l-2},\right.} \\
& \left.d_{L-1}, d_{L-2}, \ldots, d_{1}, a_{0}^{\prime}, \ldots, a_{S}^{\prime}\right] .
\end{aligned}
$$

We note that the number of partial quotients preceding $a_{0}^{\prime}$ in the previous continued fraction is $(l-1) L$. Recalling the fact that $L$ is even and the convention that first partial quotient is located in the 0th position, we see that $a_{0}^{\prime}$ appears in an even position. As $a_{0}^{\prime} \geq 3>2=d_{0}$, we may use a well known inequality involving continued fractions (see, for example, [3, Chapter 1, Lemma 1]) to conclude that

$$
\begin{aligned}
& {\left[0,\left(d_{L-1}, d_{L-2}, \ldots, d_{0}\right)_{l-2}, d_{L-1}, d_{L-2}, \ldots, d_{1}, a_{0}^{\prime}, \ldots, a_{S}^{\prime}\right]} \\
& \quad \geq\left[0,\left(d_{L-1}, d_{L-2}, \ldots, d_{0}\right)_{l-2}, d_{L-1}, d_{L-2}, \ldots, d_{1}, d_{0}, d_{L-1}, d_{L-2}, \ldots, d_{1}\right] \\
& \quad=\left[0,\left(d_{L-1}, d_{L-2}, \ldots, d_{0}\right)_{l-1}, d_{L-1}, d_{L-2}, \ldots, d_{1}\right] .
\end{aligned}
$$

Therefore identity (6.9) together with Lemma 6.4 and the fact that $C(n)$ is 
a decreasing function of $n$ yields

$$
\begin{aligned}
A_{(l-1) L-1}^{\prime} & \geq \delta_{m}+\left[0,\left(d_{L-1}, d_{L-2}, \ldots, d_{0}\right)_{l-1}, d_{L-1}, d_{L-2}, \ldots, d_{1}\right] \\
& =C(l) \geq C(n) .
\end{aligned}
$$

Hence the $n$ numbers, $A_{-1}^{\prime}, A_{L-1}^{\prime}, \ldots, A_{(n-1) L-1}^{\prime}$, are all greater than or equal to $C(n)$. By our previous remarks, this fact implies that there are at least $n$ solutions to inequality (6.1), which establishes the desired result in subcase I when $k=0$.

Next we assume that $k \geq 1$. For each $l, 1 \leq l \leq n$, we consider

$$
A_{l L-1}^{\prime}=\left[a_{l L}^{\prime}, a_{l L+1}^{\prime}, \ldots\right]+\left[0, a_{l L-1}^{\prime}, a_{l L-2}^{\prime}, \ldots, a_{S}^{\prime}\right] .
$$

Thus as we observed in the $k=0$ case, we may write

$$
\begin{aligned}
A_{l L-1}^{\prime}= & {\left[\overline{d_{0}, d_{1}, \ldots, d_{L-1}}\right] } \\
& +\left[0,\left(d_{L-1}, d_{L-2}, \ldots, d_{0}\right)_{l-1}, d_{L-1}, d_{L-2}, \ldots, d_{k+1}, a_{k}^{\prime}, \ldots, a_{S}^{\prime}\right] .
\end{aligned}
$$

As there are $l L-k$ partial quotients preceding $a_{k}^{\prime}$ in the previous continued fraction, and $L$ is even, we see that if $k$ is also even, then $a_{k}^{\prime}$ is located in an even-indexed location, and if $k$ is odd, then $a_{k}^{\prime}$ appears in an odd-indexed position. As we are in subcase I, we know that either $a_{k}^{\prime}<d_{k}$ and $a_{k}^{\prime}$ appears in an odd-indexed position, or $a_{k}^{\prime}>d_{k}$ and $a_{k}^{\prime}$ appears in an even-indexed position. Therefore, in view of a well known inequality involving continued fractions (see [3, Chapter 2, Lemma 1]), together with identity (6.3), and Lemma 6.4, we conclude

$$
\begin{aligned}
A_{l L-1}^{\prime}= & {\left[\overline{d_{0}, d_{1}, \ldots, d_{L-1}}\right] } \\
& +\left[0,\left(d_{L-1}, d_{L-2}, \ldots, d_{0}\right)_{l-1}, d_{L-1}, d_{L-2}, \ldots, d_{k+1}, a_{k}^{\prime}, \ldots, a_{S}^{\prime}\right] \\
\geq & {\left[\overline{d_{0}, d_{1}, \ldots, d_{L-1}}\right] } \\
& +\left[0,\left(d_{L-1}, d_{L-2}, \ldots, d_{0}\right)_{l-1}, d_{L-1}, d_{L-2}, \ldots, d_{k+1}, d_{k}, \ldots, d_{1}\right] \\
= & \delta_{m}+\left[0,\left(d_{L-1}, d_{L-2}, \ldots, d_{0}\right)_{l-1}, d_{L-1}, d_{L-2}, \ldots, d_{1}\right] \\
= & C(l) \geq C(n) .
\end{aligned}
$$

Thus each of the values $A_{L-1}^{\prime}, A_{2 L-1}^{\prime}, \ldots, A_{n L-1}^{\prime}$ is greater than or equal to $C(n)$, which implies that there are at least $n$ solutions to inequality (6.1). Hence we have established the result in subcase I.

We now examine subcase II; that is, either $a_{k}^{\prime}>d_{k}$ and $k$ is odd or $a_{k}^{\prime}<d_{k}$ and $k$ is even. By Lemma 6.3, $\gamma_{m}=\left[\frac{a_{J}, d_{J+1}, \ldots, d_{J+(L-1)}}{d^{\prime}}\right]=$ $\left[\overline{d_{L}, d_{L-1}, \ldots, d_{1}}\right]$ for some odd positive integer $J$. We now consider the three possibilities: $k=J, J+1 \leq k \leq L-1$, and $S \leq k \leq J-1$.

If $k=J$, then as $J$ is odd, we have $a_{J}^{\prime}>d_{J}=d_{L}=2$. That is, $a_{J}^{\prime} \geq 3$ and for all $i \geq J+1, a_{i}^{\prime}=d_{i}$. In view of Lemma 4.2, we have

$$
A_{J-1}^{\prime} \geq\left[a_{J}^{\prime}, a_{J+1}^{\prime}, \ldots\right] \geq\left[3, a_{J+1}^{\prime}, \ldots\right]>3>C(n) .
$$


More generally, for all $l, 2 \leq l \leq n$,

$$
\begin{aligned}
A_{J+(l-1) L-1}^{\prime}= & {\left[a_{J+(l-1) L}^{\prime}, a_{J+(l-1) L+1}^{\prime}, \ldots\right] } \\
& +\left[0, a_{J+(l-1) L-1}^{\prime}, a_{J+(l-1) L-2}^{\prime}, \ldots, a_{S}^{\prime}\right] \\
= & {\left[d_{J+(l-1) L}, d_{J+(l-1) L+1}, \ldots\right] } \\
& +\left[0, d_{J+(l-1) L-1}, d_{J+(l-1) L-2}, \ldots, d_{J+1}, a_{J}^{\prime}, \ldots, a_{S}^{\prime}\right],
\end{aligned}
$$

which, given that $d_{i}=d_{L+i}$, can be expressed as

$$
\begin{aligned}
A_{J+(l-1) L-1}^{\prime}= & {\left[\overline{d_{J}, d_{J+1}, \ldots, d_{J+(L-1)}}\right] } \\
& +\left[0,\left(d_{J+(L-1)}, d_{J+(L-2)}, \ldots, d_{J}\right)_{l-2},\right. \\
& \left.\quad d_{J+(L-1)}, d_{J+(L-2)}, \ldots, d_{J+1}, a_{J}^{\prime}, \ldots, a_{S}^{\prime}\right] .
\end{aligned}
$$

The number of partial quotients preceding $a_{J}^{\prime}$ in the continued fraction is $(l-1) L$. Thus as $L$ is even, $a_{J}^{\prime}$ appears in an even-indexed position and $a_{J}^{\prime} \geq 3>d_{J}$, we conclude that

$$
\begin{gathered}
{\left[0,\left(d_{J+(L-1)}, d_{J+(L-2)}, \ldots, d_{J}\right)_{l-2}, d_{J+(L-1)}, d_{J+(L-2)},\right.} \\
\left.\ldots, d_{J+1}, a_{J}^{\prime}, \ldots, a_{S}^{\prime}\right] \\
\geq\left[0,\left(d_{J+(L-1)}, d_{J+(L-2)}, \ldots, d_{J}\right)_{l-2}, d_{J+(L-1)}, d_{J+(L-2)},\right. \\
\left.\ldots, d_{J+1}, d_{J}, d_{J+(L-1)}, d_{J+(L-2)}, \ldots, d_{J+1}\right] \\
=\left[0,\left(d_{J+(L-1)}, d_{J+(L-2)}, \ldots, d_{J}\right)_{l-1}, d_{J+(L-1)}, d_{J+(L-2)}, \ldots, d_{J+1}\right] .
\end{gathered}
$$

In view of identity (6.4), Lemma 6.4, and Lemma 4.2, inequality (6.10) reveals

$$
\begin{aligned}
A_{J+(l-1) L-1}^{\prime} \geq & \gamma_{m} \\
& +\left[0,\left(d_{J+(L-1)}, d_{J+(L-2)}, \ldots, d_{J}\right)_{l-1}, d_{J+(L-2)}, \ldots, d_{J+1}\right] \\
= & C(l) \geq C(n) .
\end{aligned}
$$

Thus each of $A_{J-1}^{\prime}, A_{J+(L-1)}^{\prime}, \ldots, A_{J+(n-1) L-1}^{\prime}$ is greater than or equal to $C(n)$, which establishes the desired result in subcase II when $k=J$.

Adopting the previous arguments in the case when $J+1 \leq k \leq L-1$, it can be shown that for any $l, 1 \leq l \leq n$,

$$
\begin{aligned}
A_{J+l L-1}^{\prime} \geq & {\left[\overline{d_{J}, d_{J+1}, \ldots, d_{J+(L-1)}}\right] } \\
& +\left[0,\left(d_{J+(L-1)}, d_{J+(L-2)}, \ldots, d_{J}\right)_{l-1}, d_{J+(L-1)}, d_{J+(L-2)},\right. \\
= & \gamma_{m}+\left[0,\left(d_{J+(L-1)}, d_{J+(L-2)}, \ldots, d_{J+1}, d_{k}, \ldots, d_{J+1}\right]\right. \\
= & C(l) \geq C(n),
\end{aligned}
$$

which establishes the theorem in this case. 
Finally, if $S \leq k \leq J-1$, then it can be shown that for any $l, 1 \leq l \leq n$,

$$
\begin{aligned}
A_{J+(l-1) L-1}^{\prime} \geq & {\left[\overline{d_{J}, d_{J+1}, \ldots, d_{J+(L-1)}}\right] } \\
& \quad+\left[0,\left(d_{J+(L-1)}, d_{J+(L-2)}, \ldots, d_{J}\right)_{l-1},\right. \\
& \left.\quad d_{J+(L-1)}, d_{J+(L-2)}, \ldots, d_{k+1+L}, d_{k+L}, d_{J+1}\right] \\
= & \gamma_{m}+\left[0,\left(d_{J+(L-1)}, d_{J+(L-2)}, \ldots, d_{J}\right)_{l-1},\right. \\
= & C(l) \geq C(n),
\end{aligned}
$$

which implies the desired result for subcase II. Thus we have established the theorem for case (i), that is, for $S<0$.

We now turn our attention to case (ii), where the index $S$ satisfies $S \geq 0$. If there exists an index $i, 0 \leq i \leq L-1$, satisfying $a_{i}^{\prime} \neq d_{i}$, then we may proceed exactly as in the previous case, consider two subcases and produce the desired result. We remark that in adopting the previous argument in this situation, if $k=0$, then it would follow that $\alpha=1 / \delta_{m}$. However as we are currently only considering the case $\alpha \neq 1 / \delta_{m}$, we conclude that $k \neq 0$ when $S \geq 0$. This point is crucial since in the previous case, when $k=0$, one of our solutions arose from the $A_{-1}^{\prime}$ term, which when $S \geq 0$, would not exist. We now see, however, that when $\alpha \neq 1 / \delta_{m}$, we are never in the situation of $k=0$ and $S \geq 0$.

Thus we may now assume that for all $i \geq 0, a_{i}^{\prime}=d_{i}$. We note that if $S=0$ in this case, then we have $\alpha=1 / \delta_{m}$, which again is impossible. Therefore we see that $S>0$ and for all $i \geq S, a_{i}^{\prime}=d_{i}$.

We first consider the case when $S$ is odd. Here we can adopt the previous arguments and verify that for any $l, 1 \leq l \leq n$,

$$
\begin{aligned}
A_{l L-1}^{\prime} & =\left[\overline{d_{l L}, d_{l L+1}, d_{l L+(L-1)}}\right]+\left[\left(d_{L-1}, d_{L-2}, \ldots, d_{0}\right)_{l-1}, d_{L-1},\right. \\
& \left.d_{L-2}, \ldots, d_{S}\right] \\
& \geq \delta_{m}+\left[\left(d_{L-1}, d_{L-2}, \ldots, d_{0}\right)_{l-1}, d_{L-1}, d_{L-2}, \ldots, d_{1}\right] \\
& =C(l) \geq C(n),
\end{aligned}
$$

which implies the desired result.

Next we assume that $S$ is even and $S \geq J+1$. In this case it can be shown that for each $l, 1 \leq l \leq n$,

$$
\begin{aligned}
A_{J+l L-1}^{\prime} & =\gamma_{m}+\left[0,\left(d_{J+(L-1)}, d_{J+(L-2)}, \ldots, d_{J}\right)_{l-1}, d_{J+(L-1)},\right. \\
& \left.d_{J+(L-2)}, \ldots, d_{S}\right] \\
& \geq \gamma_{m}+\left[0,\left(d_{J+(L-1)}, d_{J+(L-2)}, \ldots, d_{J}\right)_{l-1}, d_{J+(L-1)},\right. \\
& =C(l) \geq C(n),
\end{aligned}
$$

which establishes the theorem in this case. 
Finally, if $S$ is even and $S \leq J-1$, then similar arguments show that for any $l, 1 \leq l \leq n$,

$$
\begin{aligned}
& A_{J+(l-1) L-1}^{\prime}=\gamma_{m}+\left[0,\left(d_{J+(L-1)}, d_{J+(L-2)}, \ldots, d_{J}\right)_{l-1},\right. \\
& \left.d_{J+(L-1)}, d_{J+(L-2)}, \ldots, d_{S+L}\right] \\
& \geq \gamma_{m}+\left[0,\left(d_{J+(L-1)}, d_{J+(L-2)}, \ldots, d_{J}\right)_{l-1},\right. \\
& \left.d_{J+(L-1)}, d_{J+(L-2)}, \ldots, d_{J+1}\right] \\
& =C(l) \geq C(n),
\end{aligned}
$$

which establishes the theorem in case (ii).

We now consider the special case when $\alpha=1 / \delta_{m}=\left[0, \overline{d_{0}, d_{1}, \ldots, d_{L-1}}\right]$. In this case, for any $l, 1 \leq l \leq n$, by methods employed above, we can deduce that

$$
\begin{aligned}
A_{J+(l-1) L-1} & =\left[\overline{d_{J}, d_{J+1}, \ldots, d_{J+(L-1)}}\right]+\left[\left(d_{J+(L-1)}, d_{J+(L-2)}, \ldots, d_{J}\right)_{l-1},\right. \\
& \left.d_{J+(L-1)}, d_{J+(L-2)}, \ldots, d_{L}\right] \\
& \geq \gamma_{m}+\left[0,\left(d_{J+(L-1)}, d_{J+(L-2)}, \ldots, d_{J}\right)_{l-1}, d_{J+(L-1)},\right. \\
& =C(l) \geq C(n),
\end{aligned}
$$

which yields the desired result in this last remaining case and completes the proof.

7. Proof of Theorem 1.2. Here we provide a proof of Theorem 1.2 which we state as:

TheOREM 7.1. Let $r$ and $n$ be positive integers. For an irrational number $\alpha$ not equivalent to $\alpha_{m_{s}}$ for any $s, s<r$, the inequality

$$
q\|\alpha q\| \leq \frac{1}{C_{r}(n)}
$$

is satisfied for at least $n$ positive integers $q$. Moreover, the constant $C_{r}(n)$ is best possible.

Proof. If $\alpha=\alpha_{m_{r}}$, then the result follows from Theorem 5.1. Moreover, identity (5.2) shows that $C_{r}(n)$ cannot be improved. If $\alpha \sim \alpha_{m_{r}}, \alpha \neq \alpha_{m_{r}}$, then the result follows from Theorem 6.1.

Next we consider the case when $\alpha \sim \alpha_{m_{t}}$ for some $t>r$. By Lemma 4.2 and the remarks that follow it, we have $C_{t}(n)>C_{r}(n)$ for all $n$. Thus in view of Theorems 5.1 and 6.1 , there are at least $n$ rational solutions $p / q$ to

$$
\left|\alpha-\frac{p}{q}\right| \leq \frac{1}{C_{t}(n) q^{2}}<\frac{1}{C_{r}(n) q^{2}},
$$

which establishes the desired result in this case. 
Finally we assume that $\alpha$ is not equivalent to any $\alpha_{m_{t}}$. Thus the Markoff constant for $\alpha$ satisfies $\mu(\alpha) \geq 3$. It follows from Lemma 4.2 that $C_{r}(n)<3$, for any $r$ and $n$. Hence,

$$
\left|\alpha-\frac{p}{q}\right|<\frac{1}{\mu(\alpha) q^{2}}<\frac{1}{C_{r}(n) q^{2}}
$$

has infinitely many rational solutions $p / q$, which completes our proof.

\section{References}

[1] E. B. Burger, Exploring the Number Jungle: A Journey into Diophantine Analysis, AMS Student Math. Library Ser., Providence, 2000.

[2] J. W. S. Cassels, An Introduction to Diophantine Approximation, Cambridge Univ. Press, Cambridge, 1957.

[3] T. W. Cusick and M. E. Flahive, The Markoff and Lagrange Spectra, Amer. Math. Soc., Providence, 1989.

[4] L. C. Eggan, On Diophantine approximations, Trans. Amer. Math. Soc. 99 (1961), $102-117$.

[5] A. Hurwitz, Über die angenäherte Darstellung der Irrationalzahlen durch rationale Brüche, Math. Ann. 39 (1891), 279-284.

[6] A. Markoff, Sur les formes quadratiques binaires indéfinies, ibid. 15 (1879), 381-406.

[7] A. V. Prasad, Note on a theorem of Hurwitz, J. London Math. Soc. 23 (1948), 169-171.

[8] M. Prasad and K. C. Prasad, A note on Diophantine approximation, Proc. Edinburgh Math. Soc. (2) 18 (1972/73), 137-142.

[9] J. Tong, The constant for finite Diophantine approximation, Fibonacci Quart. 35 (1997), 29-31.

Department of Mathematics

Williams College

Williamstown, MA 01267, U.S.A.

E-mail: Edward.B.Burger@williams.edu

Received on 12.2.2001

and in revised form on 11.6.2001 